\title{
Cyclohexanohemicucurbit[8]uril forms inclusion complexes with heterocycles and removes sulfuric guests from water via solid-phase extraction
}

Tatsiana Shalima ${ }^{a}$, Kamini A. Mishra ${ }^{a}$, Sandra Kaabel ${ }^{b}$, Lukas Ustrnul ${ }^{a}$, Simona Bartkova ${ }^{a}$, Kaia Tõnsuaadu ${ }^{c}$, Ivo Heinmaa ${ }^{d}$ and Riina Aav*a

a. Department of Chemistry and Biotechnology, Tallinn University of Technology, Akadeemia tee 15, 12618, Tallinn, Estonia, E-mail: riina.aav@taltech.ee

b. Department of Chemistry, McGill University, 801 Sherbrooke St. West, Montréal, Québec H3A OB8, Canada.

c. Laboratory of Inorganic Materials, Institute of Materials and Environmental Technology, Tallinn University of Technology, Ehitajate tee 5, 12616, Tallinn, Estonia.

d. Laboratory of Chemical Physics, National Institute of Chemical Physics and Biophysics, Akadeemia tee 23, 12618, Tallinn, Estonia

Supramolecular chemistry, in particular, molecular recognition ${ }^{1,2}$, sensing ${ }^{3-5}$ and transport $^{6-9}$ have promoted extensive research toward new applications. Out of many synthetic receptors, macrocycles with confined inner spaces demonstrate the specific hostguest interaction via inclusion complex formation. ${ }^{2,10-17}$. Hemicucurbiturils formed in templated single step oligomerization reactions, ${ }^{18}$ are single-bridged cucurbituril-type macrocycles ${ }^{19-21}$ that bear an electron-deficient hydrophobic cavity. The latter grants these macrocycles the ability to encapsulate anions, ${ }^{19,18,21-32}$, and also the formation of complexes with acids and some neutral species has been reported. In particular, unsubstituted hemicucurbit $[n]$ urils $(n=6,12)$ bind phenol derivatives ${ }^{33,34}$ and ferrocene, ${ }^{35}$ and cyclohexanohemicucurbit $[n]$ urils $(\mathrm{cycHC}[n])(n=6,8,12)^{36-40}$ form external complexes with inorganic and organic acids. ${ }^{41,42,38,39}$ However, the binding of heterocycles by hemicucurbiturils has not been thoroughly studied. We envisioned that heterocycles 1-13 have relatively high electron density compared to carbocycles and may be able to occupy space within the eightmembered cycHC[8] (Fig. 1). S-heterocycles are compounds of interest as these occur naturally 43 and contribute to the distinct aroma of food, ${ }^{44,45}$ furthermore, tetrahydrothiophene $\mathbf{2}$ is added as an odorant to natural gas. Unsubstituted S-containing heterocycles, such as 1,3-dithiolane 1 and 1,4-thioxane 3, are found in meat ${ }^{46}$ and around chemical warfare dumping sites, some located in the Baltic Sea, where they are formed due to degradation of mustard gas. ${ }^{47-49}$ Many sulfur compounds bearing Sheterocycle fragments are bioactive. ${ }^{50,51}$ For instance, $\alpha$-lipoic acid $\mathbf{9}$ exhibits antioxidative properties and is used as a dietary supplement and a pharmaceutical agent. ${ }^{52,53}$ Thus far, cyclodextrins have been applied to form complexes with $\alpha$-lipoic acid, which is hydrophobic by nature, to enhance its solubility and bioavailability. ${ }^{54-60}$

Commercially available solid-phase extraction (SPE) tubes ${ }^{61}$ and novel sorbent materials ${ }^{62}$ are used for the removal of S-heterocycles from water; however, such systems are designed to non-selectively retain all non-polar to moderately polar components. At the same time, to supplement the non-specific materials, selective sorbents can be employed.

Herein, we report that $\mathrm{cycHC}[8]$ encapsulates neutral electron-rich organic molecules, namely, five- and six-membered heterocycles containing sulfur and oxygen atoms. This binding was characterized by ${ }^{1} \mathrm{H}$ NMR titration and isothermal titration calorimetry (ITC) in solution, and the structure of complexes was investigated by single crystal X-ray diffraction (SC-XRD), solidstate NMR (ssNMR), and thermogravimetric analysis (TGA). The solid-phase extraction study found that cycHC[8] is a promising sorbent material.

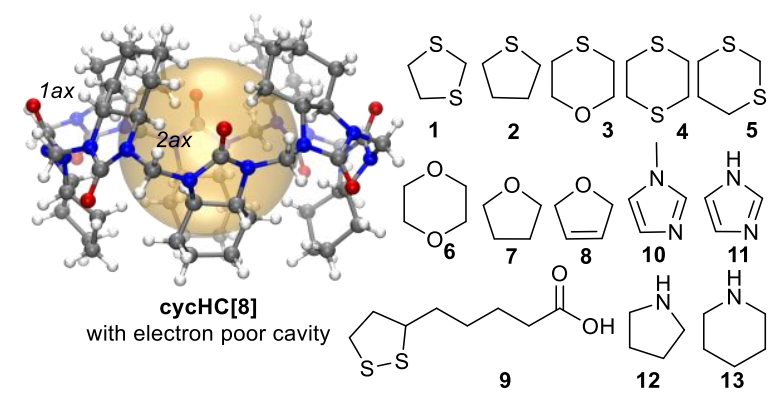

Figure 1. The structure of $\mathrm{cycHC}[8]$, highlighting the electron poor cavity for guest binding and characteristic atom numbers, and the scope of studied guests.

A series of suitably sized electron-rich S-, O- and $\mathrm{N}$-heterocyclic compounds were crystallized via slow evaporation, and compounds 1, 3, 6, 7 and 8 formed inclusion complexes with $(R, R)$-cycHC[8] upon co-crystallization (Fig. 2 and S4-S13, ESI). The N-containing heterocycles, 12 and 13, and the largest explored guest, 4, did not yield crystals of inclusion complexes with cycHC[8]. The packing of the $\mathbf{1}$ and $\mathbf{3}$ inclusion complexes with cycHC[8] gave rise to isomorphous $\left(Z^{\prime}=4\right)$ crystal structures (Fig. S4-S5, ESI), in an arrangement previously unrecorded for $\mathrm{cycHC}[8]$ inclusion complexes. The packing of complexes involving O-containing smaller heterocycles $\mathbf{6}, \mathbf{7}, \mathbf{8}$ appears to be mainly directed by hydrogen bonding interactions with methanol so that the resulting crystal structures are isomorphous to each other (Fig. S1-S3, ESI) and the previously published methanol solvate of $(R, R)$-cycHC[8]. ${ }^{38}$ However, clear features in the electron density map within the macrocycles in the respective host-guest complexes allowed to resolve the orientation of the encapsulated guests (Fig. S6-S7, ESI). The smaller guest molecules 6, 7, and 8, appear more 
disordered within $\mathrm{cycHC}[8]$, with a total site occupancy of the resolved disorder components limited to 50-75\%. The remaining electron density map had no clear features, making it impossible to resolve whether the diffuse component of the guest disorder also includes partial substitutional disorder from methanol. In contrast, the position of the larger S-containing guests, $\mathbf{1}$ and $\mathbf{3}$, is more conserved within the respective crystal structures (Fig. S6-S7, ESI) with almost no diffuse component observed, indicating that these guests have fewer orientations available within the $\mathrm{cycHC}$ [8]. Notably, analyzing the disorder models of all inclusion complexes reveals a prevalent motif, where guests are oriented with heteroatoms close to the portals of cycHC[8] (Fig. 2, see ESI for full details). These are in several structures at a suitable distance (2.7-2.8 $\mathrm{A}$ ) from a methanol molecule located at the portal of $\mathrm{cycHC}[8]$ and can therefore potentially accept hydrogen bonds through the portals of the macrocycle. The latter would explain the observed conservation of this type of guest orientation motif. The guest molecules are tightly enwrapped within the cycHC[8], especially the largest S-containing $\mathbf{1}$ and $\mathbf{3}$ that fill close to $70 \%$ of the cavity volume (Fig. 2, complexes D and E), indicating that guest binding and release must be accompanied with opening and closing the host portals. Similar conformational dynamics of $\mathrm{cycHC}[8]$ have been noted and computationally described previously in the binding of large anionic guests. ${ }^{26}$

Further, we evaluated host-guest complex formation in $\mathrm{CD}_{3} \mathrm{OD}$ solution. Inclusion complex formation was followed by the chemical shift change of $\mathrm{cycHC}$ [8] proton H2ax (Fig. 1, ESI S14-S15), positioned inside the cavity and becoming magnetically deshielded upon binding with the electron-rich guest. The studies revealed that S-containing five-membered heterocycles, 1 and 2 caused a larger chemical shift change of 0.064 and 0.048 ppm, respectively, compared to the six-membered heterocycles; 3, 4, $\mathbf{5}$ and $\mathbf{6}$. This can be explained by a higher affinity and a larger population of complexed species formed with five-membered Sheterocycles. 1 induced the most prominent shift in the $\mathrm{H} 2 \mathrm{ax}$ signal of $\mathrm{cycHC}[8]$ among all the compounds studied. A negligible shift was observed for 7, while no shift was observed for $\mathbf{8}$ and $\mathbf{N}$-heterocycles, 10 and 11. Signals of 9 overlapped with the characteristic cycHC[8] signal, and therefore its encapsulation could not be evaluated by ${ }^{1} \mathrm{H}$ NMR. The differences in the affinity of guests for binding to $\mathrm{cycHC}[8]$ are seemingly dependent on the hydrophobicity of the guests, which can be characterized by their partition coefficients (Table S6, ESI). Nevertheless, all chemical shift changes were relatively small compared to those occurring upon the binding of anions ( $0.6 \mathrm{ppm}$ in the $\mathrm{H} 2 \mathrm{ax}$ signal). ${ }^{26}$ The binding of 1, was further evaluated by NMR titration, and a weak association constant, $K=7.9 \pm 0.2 \mathrm{M}^{-1}$, was determined by a one-to-one binding model (Fig. 3, S16-S17, ESI). ITC titration was performed to evaluate the thermodynamic parameters of binding $\mathbf{3}$ and $\mathbf{1}$ (Fig. 3, Table 1, S18-S20, ESI). The Gibbs free energy and association constant values for binding in $\mathrm{CH}_{3} \mathrm{OH}$ show that 1 interacts with cycHC[8] more strongly than 3, resulting in the association constants $13.1 \mathrm{M}^{-1}$ and $2.5 \mathrm{M}^{-1}$, respectively. Consequently, the binding of other $\mathrm{S}$ - and O-containing guests is expected to fall below the binding constant of $\mathbf{1}$. The binding of both guests in methanol was enthalpically favorable and entropically unfavorable. A similar binding character was also observed upon the binding of chaotropic anions to cycHC[8] in protonic media. ${ }^{26}$ Even though chaotropicity is mainly attributed to the ionic species, there have been reports of chaotrope-like organic molecules studied in crystalline hydrates. ${ }^{63}$ As chaotropic character ${ }^{23}$ is exhibited in aqueous media, we further studied the binding of 1 with cycHC[8] in a 1:1 mixture of $\mathrm{CH}_{3} \mathrm{OH}$ and $\mathrm{H}_{2} \mathrm{O}$. The results showed that the presence of water enhances the binding of the guest, raising the association constant to $65.6 \mathrm{M}^{-1}$. The enthalpy of binding was strongly increased in the presence of water, accompanied by a rise of the entropic penalty (Table 1 ). However, such binding study in pure water was not possible due to the very low watersolubility of $\mathrm{cycHC}[8]$.

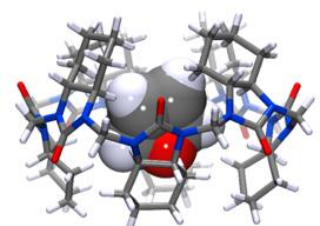

A

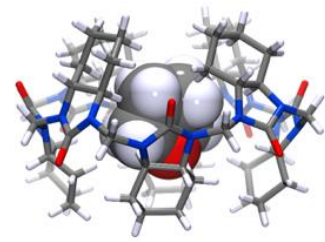

B

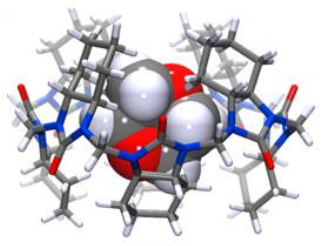

C

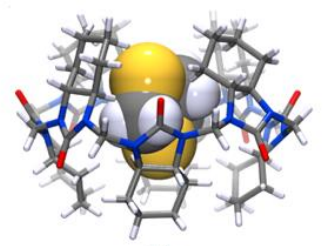

D

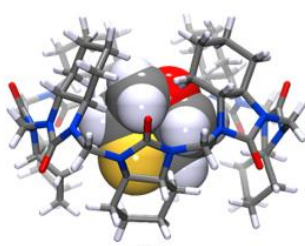

$\mathbf{E}$

Figure 2. Crystal structures of cycHC[8] inclusion complexes with neutral heterocycles with increasing packing coefficient order: 8@cycHC[8] A, (PC 0.51), 7@cycHC[8] B, (PC 0.54), 6@cycHC[8] C (PC 0.61), 1@cycHC[8] D (PC 0.66), 3@cycHC[8] E (PC 0.69) and their Packing Coefficient value is the ratio between $V_{\text {guest }}$ to $V_{\text {cavity }}($ host), reflecting the space filled by the encapsulated guest in the host cavity. ${ }^{64}$ For more details, see Table S2, ESI. 

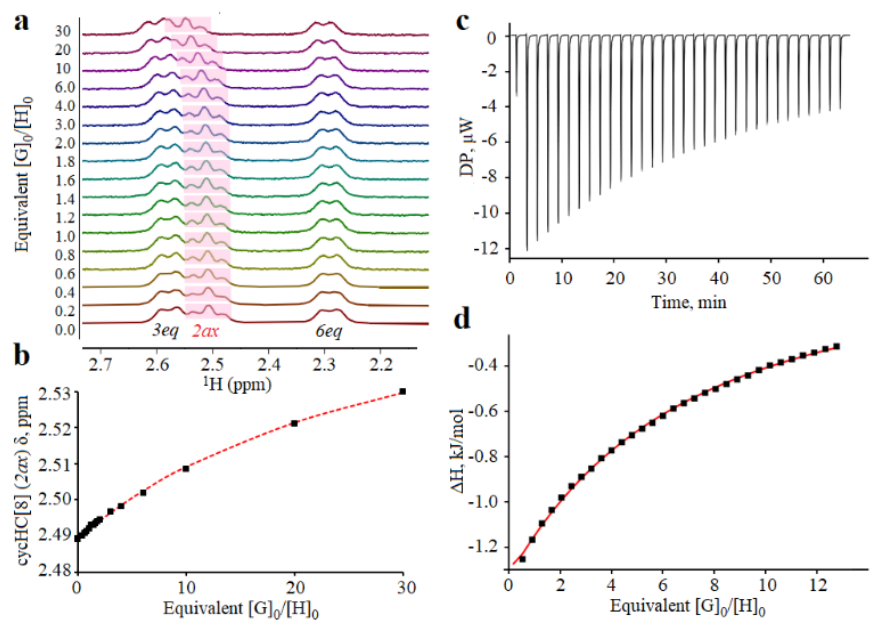

Figure 3. ${ }^{1} \mathrm{H}$ NMR and ITC titration for cycHC[8] binding with 1. a) Spectra for NMR titration in $\mathrm{CD}_{3} \mathrm{OD}$, b) Binding isotherm for NMR titration, 1:1 binding model, c) Raw thermogram for ITC measurement in 1:1 $\mathrm{CH}_{3} \mathrm{OH}: \mathrm{H}_{2} \mathrm{O}$ mixture, d) Binding isotherm for ITC measurement using the "one set of sites" model.

Table 1. Thermodynamic parameters from ITC measurements for complexation of guest 1 and 3 with cycHC[8] for the $1: 1$ binding model. All energy values are given in kJ/mol.

\begin{tabular}{cccccc}
\hline Guest & Solvent & $\boldsymbol{\Delta} \boldsymbol{H}^{\circ}$ & $-\boldsymbol{T} \boldsymbol{\Delta} \boldsymbol{S}^{\circ}$ & $\boldsymbol{\Delta} \boldsymbol{G}^{\circ}$ & $\boldsymbol{K}, \mathbf{M}^{-\mathbf{1}}$ \\
\hline $\mathbf{3}$ & $\mathrm{CH}_{3} \mathrm{OH}$ & $-13.7 \pm 1.2$ & 11.4 & -2.3 & $2.5 \pm 0.2$ \\
\hline \multirow{2}{*}{$\mathbf{1}$} & $\mathrm{CH}_{3} \mathrm{OH}$ & $-9.8 \pm 0.6$ & 3.6 & -6.2 & $13.1 \pm 0.8$ \\
& $\begin{array}{c}\mathrm{CH}_{3} \mathrm{OH}: \mathrm{H}_{2} \mathrm{O} \\
(1: 1)\end{array}$ & $-20.4 \pm 0.9$ & 10.2 & -10.2 & $65.6 \pm 2.5$ \\
\hline
\end{tabular}

The low solubility of $\mathrm{cycHC}[8]$ in water and its ability to form inclusion complexes with heterocycles encouraged us to study the removal of S-containing heterocycles from water via solid-phase extraction (SPE). ). To evaluate whether the ability of cycHC[8] to form inclusion complexes influences the sorption of heterocycles, we performed extraction experiments in parallel with the 6-membered homolog ( $\mathrm{cycHC}[6])$ of $\mathrm{cycHC}[8]$. The $\mathrm{cycHC}[6]$ consists of the same monomers, so the hydrophobic properties of the outer surface are very similar to cycHC[8], but its cavity is much smaller $\left(35 \AA^{3}\right), 38$ and thus it cannot accommodate the heterocycles studied. Hence, it served as a very informative analog for determining external or internal binding during extraction. $\mathrm{CycHC}[6]$ is also very poorly soluble in water and can be used in SPE as a solid sorbent. Textural properties of the sorbents, such as particle size and surface area, are important for the binding efficacy; therefore, prior to extraction experiments, the macrocyclic hosts were milled to obtain finer powders. Microscopic studies and image analysis of the samples (S21-S24, ESI) before and after milling showed that milling of both macrocycles produced particles of relatively uniform size, eliminating the larger aggregates and resulting in ca $80 \%$ of particles having a diameter under $5 \mu \mathrm{m}$. In addition, the Brunauer-Emmett-Teller (BET) surface area of the solid extraction material was investigated by $\mathrm{N}_{2}$ adsorption-desorption analysis (S25-S26, ESI) and was found to be $6.03 \mathrm{~m}^{2} / \mathrm{g}$ and $9.02 \mathrm{~m}^{2} / \mathrm{g}$ for $\mathrm{cycHC}[6]$ and $\mathrm{cycHC}[8]$, respectively. According to this analysis of the sorbent material, and in the case where selectivity of binding would be caused by the availability of the surface area, $\mathrm{cycHC}[n]$ homologs are expected to have similar extraction efficiencies.

SPE was performed for heterocycles $\mathbf{1}, \mathbf{3}, \mathbf{6}, \mathbf{9}, \mathbf{1 0}$ and 11, by stirring solid $\mathrm{cycHC}[n]$ in an aqueous solution of each guest, and the change in the guest concentration was analyzed upon extraction. This change was assumed to be equal to the amount of hostguest complex formed and is expressed as sorption efficiency in Fig. 4a. The results (Fig. 4a, Table S7, ESI) demonstrate that roughly 5 molar excess of $\mathrm{cycHC}[n]$ gave negligible removal of O-containing 6 with cycHC[8] (5\%) and was inefficient in the case of cycHC[6], as well as for both macrocycles in the case of the $\mathbf{N}$-containing $\mathbf{1 0}$ and $\mathbf{1 1}$ (Table S13, ESI). In contrast, cycHC[8] can efficiently extract the S-containing 1 (78 \%) and moderately remove 3 (25\%), while cycHC[6] was considerably less efficient at removing these guests, $16 \%$ for $\mathbf{1}$ and only $3 \%$ of $\mathbf{3}$. These results indicate that the ability of cycHC[8] to form inclusion complexes with heterocycles enhances its extraction ability. Also, the extraction efficiency of cycHC[8] correlates with a stronger affinity toward Scontaining heterocycles observed in our solution studies above. The reusability of the sorbent after binding of 1 was studied (Fig. $4 \mathrm{~b})$, and it was found that the solid macrocyclic material, cycHC[8], can be used multiple times. A simple washing and drying procedure, followed by milling, allows the reactivation of the sorbent's surface for future use without significant loss in binding efficiency for at least four cycles (Fig. 4a, Table S14, ESI). ) It was also confirmed that cycHC[8] was recovered with sufficient purity (Fig. S48). In order to confirm that $\mathrm{cycHC}$ [8] can accommodate S-containing heterocycles in its cavity during SPE, thermogravimetric analysis of pure 1 and of $\mathrm{cycHC}[n]$ sorbents enriched with extracted $\mathbf{1}$ was undertaken (S37, ESI). Firstly, it was confirmed that the cycHCs exhibit thermal stability upon heating up to $200^{\circ} \mathrm{C}$, and no decomposition was observed. For the investigation of hostguest binding, solid macrocyclic $\mathrm{cycHC}[n]$, which retained guest molecules after the extraction procedure, were analyzed in parallel with pure 1. At a certain temperature, pure 1 would start to partially decompose, releasing volatile degradation products. The 
formation of characteristic mass-fragments of 1 with $\mathrm{m} / \mathrm{z} 47$, corresponding to $\left[\mathrm{CH}_{2} \mathrm{SH}\right]^{-}$was followed during this analysis. The evolution profiles for pure 1 and its host-guest complex with cycHC[6] are similar (Fig. 5a), indicating the decomposition products were released within the same temperature range and producing maximum ion current at $148^{\circ} \mathrm{C}$ and $138^{\circ} \mathrm{C}$, respectively. Meanwhile, the complex with $\mathrm{cycHC}[8]$ releases the characteristic degradation product at a significantly higher temperature, $189{ }^{\circ} \mathrm{C}$ (Fig. 5a and Fig. S40A, ESI). This is regarded as evidence of 1 encapsulation into the cycHC[8] cavity during the extraction procedure, which leads to a higher affinity toward this guest.

The results of the extraction of the larger and more hydrophobic 9 are much more similar between $\mathrm{cycHC}[6]$ and $\mathrm{cycHC}[8]$ (Fig. 4a). With 5 eq. cycHC[8], $74 \%$ of 9 was removed from the aqueous solution.
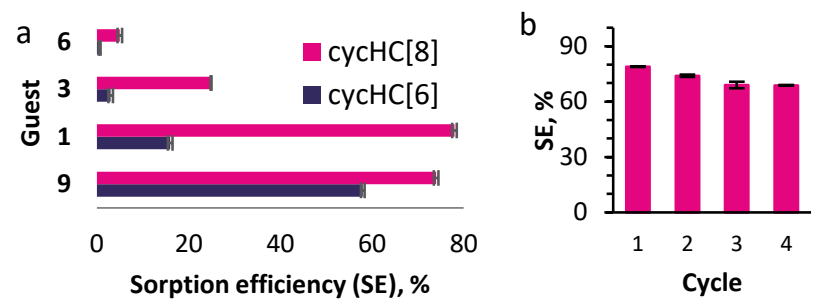

Figure 4. a) Sorption efficiency (SE) of SPE of neutral guests from water conducted in presence of 4-6 eq. of cycHC[6] and cycHC[8] (dark blue and pink, respectively) for 6, 3, 1 and 9. b) Sorption efficiency of $\mathrm{cycHC}[8]$ during four 1 extraction cycles. (error bars are the standard deviation between parallel experiments ( $n \geq 2$ for (a) and $n=3$ for $(b))$.

A higher excess (20 eq.) of the macrocyclic host, cycHC[8], resulted in the removal of over $90 \%$ of the 9 from the water, but unfortunately, this occurred with no enantioselectivity (Tables S8-S9, ESI). In a comparative study with 5 eq. excess of cycHC[6], $58 \%$ of 9 was extracted, which differs only by ca $16 \%$ for the relative efficiency gained by cycHC[8] under similar conditions. This relatively small selectivity difference indicates the very different character of $\mathbf{9}$ binding to cycHCs, compared to $\mathbf{1}$. To better understand the interaction of $\alpha$-lipoic acid $\mathbf{9}$ with $\mathrm{cycHC}[8]$ and $\mathrm{cycHC}[6]$, complex formation was studied by solid-state $\mathrm{NMR}$ (sSNMR) spectroscopy. Notably, complex formation with cucurbit[7]uril via mechanochemical agitation followed by ssNMR has also been demonstrated by Masson and Kaleta. ${ }^{65}$ The $\mathrm{cycHC}[n]$ and 9 in a 1:1 molar ratio were milled together in the presence of a small amount of water $(\eta=0.3 \mu \mathrm{l} / \mathrm{mg})$, that is, modelling the binding during the extraction process at the solute-solid interphase. The milled sample obtained and a mixture of $\mathbf{9}$ and $\mathrm{cycHC}[n]$ stirred together without applying additional mechanical force were investigated by ${ }^{13} \mathrm{C}$ ssNMR spectroscopy (S38-S40, ESI). Fig. 5b illustrates the changes induced upon binding 9 with cycHC[8] and cycHC[6] (Fig. S43-S44, S47, ESI). Overall, 9 has clearly visible sharp signals in the range of 32-42 ppm in the sample, which was not milled, while in the milled sample, these signals were broadened, suggesting a change in the close environment of the 9 either due to interaction with the water or the $\mathrm{cycHC}$ [8]. Additionally, the carboxylic group signal of C10' shifts from $183 \mathrm{ppm}$ to $175 \mathrm{ppm}$ $\left(\mathrm{C}^{\prime \prime} 0^{\prime \prime}\right)$, indicating additional shielding of the carboxyl groups, which may be caused by disaggregation of 9 and complexation with $\mathrm{cycHC}[8]$. All eight monomers of $\mathrm{cycHC}[8]$ are symmetry-related, and though they have the same chemical shifts, for this reason, changes in chemical shifts of $\mathrm{cycHC}[8]$ are hard to notice upon the binding of 1 equivalent of $\mathbf{9}$. Very similar changes in the spectra of 9 and cycHC[6] (Fig. 5b and Fig. S46-S47, ESI) were observed in samples prepared similarly to those described above. Therefore, based on this data, we propose that the interaction between 9 and cycHC[n]s occurs from the outside surface of the macrocycles. The small difference in the extraction efficiency is caused by their small particle size and surface area differences.

a

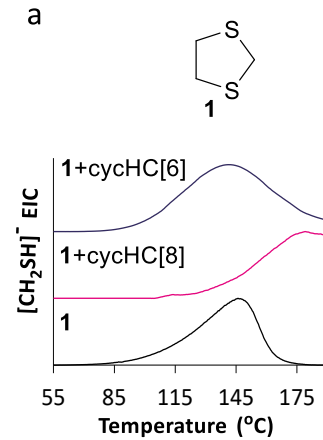

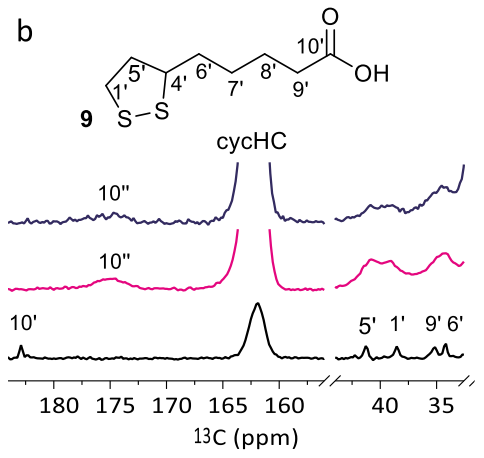

Figure 5 a) TGA analysis of $\mathbf{1}$ (lower), cycHC[8] after extraction of $\mathbf{1}$ (middle), cycHC[6] after extraction of $\mathbf{1}$; EIC stands for extracted ion current; b) ${ }^{13} \mathrm{C}$ NMR spectra of $1: 1$ mixture of 9 and cycHC [8] mixed (lower), 9 and cycHC[8] milled (middle) and 9 and cycHC[6] milled (upper), with atom numbers. 
In conclusion, encapsulation of 5- and 6-membered electron-rich heterocyclic guests is mostly related to their size, which must be complementary with the host cavity, and the hydrophobic effect is one of the driving forces of the interaction. From the binding study of 1 with $\mathrm{cycHC}^{8}$ ] in methanol and a methanol-water mixture, it was evident that complex formation is even more favourable in presence of water and the thermodynamic characteristics resembled the binding of chaotropic anions. Since the $\mathrm{cycHC}[n]$ are almost insoluble in water, we showed that they can serve as solid sorbent material for SPE of heterocycles from aqueous solutions. The sulfur-containing heterocycles are found in pollutants, food aroma compounds, and bioactive substances; therefore, developing procedures for their selective removal from water is desirable. CycHC[8] was successfully applied for selective extraction of $\mathbf{1}$ via inclusion complex formation, which was proven by TGA experiments. In the case $\mathbf{9}$, the evidence collected by ${ }^{13} \mathrm{C}$ solid-state NMR supported hypothesis of an external complex with both cycHC[6] and cycHC[8], and its extraction results suggest that the binding efficiency correlated with the small differences in particle size and surface area. Crucially, the solid $\mathrm{cycHC}[8]$ sorbent material can be reused, which makes it a candidate for applications in selective SPE systems or the removal of target compounds from water, based on molecular recognition properties.

This work was supported by Estonian Research Council (PRG399, MOBJD556 and MOBJD592), the ERDF (CoE 2014-2020.4.01.150013, and CoE TK134), H2020-FETOPEN 828779 (INITIO). The authors would also like to thank Elina Suut and Jagadeesh Varma Nallaparaju for the synthesis of $\mathrm{cycHC}[n]$, Heidi Lees, and Piia Jõul for providing the S-containing heterocyclic compounds used for crystallization and extraction experiments, and Mai Uibu for BET analysis

\section{References}

1 C.-L. Deng, S. L. Murkli and L. D. Isaacs, Chem. Soc. Rev., 2020, 49, 7516-7532.

2 S. J. Barrow, S. Kasera, M. J. Rowland, J. del Barrio and O. A. Scherman, Chem. Rev., 2015, 115, $12320-12406$.

3 R. Pinalli, A. Pedrini and E. Dalcanale, Chem. Soc. Rev., 2018, 47, 7006-7026.

4 R. Kubota, Y. Sasaki, T. Minamiki and T. Minami, ACS Sens., 2019, 4, 2571-2587.

5 T. L. Mako, J. M. Racicot and M. Levine, Chem. Rev., 2019, 119, 322-477.

6 J. Mayr, C. Saldías and D. D. Díaz, Chem. Soc. Rev., 2018, 47, 1484-1515.

7 A. S. Braegelman and M. J. Webber, Theranostics, 2019, 9, 3017-3040.

8 J. T. Davis, P. A. Gale and R. Quesada, Chem. Soc. Rev., 2020, 49, 6056-6086.

9 J. Liu, X. Ding, Y. Fu, C. Xiang, Y. Yuan, Y. Zhang and P. Yu, Eur. J. Med. Chem., 2021, 212, 113105.

10 Z. Liu, S. K. M. Nalluri and J. F. Stoddart, Chem. Soc. Rev., 2017, 46, 2459-2478.

11 J. Phatsawee, O. Noriko and L. Thorsteinn, Int. J. Pharm, 2018, 535, 274-284.

12 M. Majd, M. Yazdanpanah, M. R. Bayatloo and S. Nojavan, Talanta, 2021, 229, 122296.

13 Md. T. Sikder, Md. M. Rahman, Md. Jakariya, T. Hosokawa, M. Kurasaki and T. Saito, Chem. Eng. J., 2019, 355, $920-941$.

14 G. Crini, Chem. Rev., 2014, 114, 10940-10975.

15 R. Kumar, A. Sharma, H. Singh, P. Suating, H. S. Kim, K. Sunwoo, I. Shim, B. C. Gibb and J. S. Kim, Chem. Rev., 2019, 119, 9657-9721.

16 E. Masson, X. Ling, R. Joseph, L. Kyeremeh-Mensah and X. Lu, RSC Adv., 2012, 2, 1213-1247.

17 K. I. Assaf and W. M. Nau, Chem. Soc. Rev., 2014, 44, 394-418.

18 S. Kaabel and R. Aav, Isr. J. Chem., 2018, 58, 296-313.

19 N. N. Andersen, M. Lisbjerg, K. Eriksen and M. Pittelkow, Isr. J. Chem., 2018, 58, 435-448.

20 Y. Xi, C. Man, W. Fang, J. Xian-Yi, C. Hang and T. Zhu, Mini-Rev. Org. Chem., 2018, 15, $274-282$.

21 T. Lizal and V. Sindelar, Isr. J. Chem., 2018, 58, 326-333.

22 O. Reany, A. Mohite and E. Keinan, Israel Journal of Chemistry, 2018, 58, 449-460.

23 K. I. Assaf and W. M. Nau, Angew. Chem. Int. Ed, 2018, 57, 13968-13981.

24 H.-J. Buschmann, E. Cleve and E. Schollmeyer, Inorg. Chem. Commun., 2005, 8, 125-127.

25 E. I. Cucolea, H.-J. Buschmann and L. Mutihac, Supramol. Chem., 2016, 28, 727-732.

26 S. Kaabel, J. Adamson, F. Topić, A. Kiesilä, E. Kalenius, M. Öeren, M. Reimund, E. Prigorchenko, A. Lõokene, H. J. Reich, K. Rissanen and R. Aav, Chem. Sci., 2017, 8, 2184-2190.

27 J. Vázquez and V. Sindelar, Chem. Commun., 2018, 54, 5859-5862.

28 M. Kandrnálová, Z. Kokan, V. Havel, M. Nečas and V. Šindelář, Angewandte Chemie International Edition, $2019,58,18182-18185$.

29 N. N. Andersen, K. Eriksen, M. Lisbjerg, M. E. Ottesen, B. O. Milhøj, S. P. A. Sauer and M. Pittelkow, J. Org. Chem., $2019,84,2577-2584$.

30 H. Valkenier, O. Akrawi, P. Jurček, K. Sleziaková, T. Lízal, K. Bartik and V. Šindelář, Chem, 2019, 5, 429-444.

31 K. Maršálek and V. Šindelář, Org. Lett., 2020, 22, 1633-1637.

32 J. Sokolov, A. Štefek and V. Šindelář, ChemPlusChem, 2020, 85, 1307-1314.

33 X.-Y. Jin, F. Wang, H. Cong and Z. Tao, J. Incl. Phenom. Macrocycl. Chem., 2016, 86, $249-254$.

34 X.-Y. Jin, F. Wang, H. Cong and Z. Tao, J. Incl. Phenom. Macrocycl. Chem., 2016, 86, 241-248.

35 X.-Y. Jin, J.-L. Zhao, F. Wang, H. Cong and Z. Tao, J. Organomet. Chem., 2017, 846, 1-5.

36 Y. Li, L. Li, Y. Zhu, X. Meng and A. Wu, Cryst. Growth Des., 2009, 9, 4255-4257.

37 R. Aav, E. Shmatova, I. Reile, M. Borissova, F. Topić and K. Rissanen, Org. Lett., 2013, 15, 3786-3789.

38 E. Prigorchenko, M. Öeren, S. Kaabel, M. Fomitšenko, I. Reile, I. Järving, T. Tamm, F. Topić, K. Rissanen and R. Aav, Chem. Commun., 2015, 51, 10921-10924. 
39 E. Prigorchenko, S. Kaabel, T. Narva, A. Baškir, M. Fomitšenko, J. Adamson, I. Järving, K. Rissanen, T. Tamm and R. Aav, Chem. Commun., 2019, 55, 9307-9310.

40 K. A. Mishra, J. Adamson, M. Öeren, S. Kaabel, M. Fomitšenko and R. Aav, Chem. Commun., 2020, 56, 14645-14648.

41 M. Öeren, E. Shmatova, T. Tamm and R. Aav, Phys. Chem. Chem. Phys., 2014, 16, 19198-19205.

42 L. Ustrnul, S. Kaabel, T. Burankova, J. Martõnova, J. Adamson, N. Konrad, P. Burk, V. Borovkov and R. Aav, Chem. Commun., 2019, 55, 14434-14437.

43 K. Mahadevan and L. Farmer, J. Agric. Food Chem., 2006, 54, 7242-7250.

44 D. S. Mottram and H. R. Mottram, in Heteroatomic Aroma Compounds, ACS, 2002, vol. 826, pp. 73-92.

45 S. Schoenauer and P. Schieberle, J. Agric. Food Chem., 2018, 66, 4189-4199.

46 V. Garbusov, G. Rehfeld, G. Wölm, R. V. Golovnja and M. Rothe, Mol. Nutr. Food. Res., 1976, 20, 235-241.

47 B. T. Røen, E. Unneberg, J. Aa. Tørnes and E. Lundanes, J. Chromatogr. A, 2010, 1217, 2171-2178.

48 R. Magnusson, T. Nordlander and A. Östin, J. Chromatogr. A, 2016, 1429, 40-52.

49 M. Söderström, CHEMSEA WP3. Summary of Chemical Analysis of Sediment Samples, http://chemsea.eu/(accsessed 16.04.2021), 2014.

50 T. Rezanka, M. Sobotka, J. Spizek and K. Sigler, Antiinfect. Agents Med. Chem, 2006, 5, 187-224.

51 C. Lamberth, H. Walter, F. M. Kessabi, L. Quaranta, R. Beaudegnies, S. Trah, A. Jeanguenat and F. Cederbaum, Phosphorus. Sulfur. Silicon Relat. Elem., 2015, 190, 1225-1235.

52 L. Rochette, S. Ghibu, C. Richard, M. Zeller, Y. Cottin and C. Vergely, Mol. Nutr. Food Res., 2013, 57, 114-125.

53 B. Salehi, Y. Berkay Yılmaz, G. Antika, T. Boyunegmez Tumer, M. Fawzi Mahomoodally, D. Lobine, M. Akram, M. Riaz, E. Capanoglu, F. Sharopov, N. Martins, W. C. Cho and J. Sharifi-Rad, Biomolecules, 2019, 9, 356.

54 T. Lin-Hui, P. Zheng-Zhi and Y. Ying, J. Incl. Phenom. Macrocycl. Chem., 1995, 23, 119-126.

55 H. Maeda, T. Onodera and H. Nakayama, J. Incl. Phenom. Macrocycl. Chem., 2010, 68, 201-206.

56 H. Takahashi, Y. Bungo and K. Mikuni, Biosci. Biotechnol. Biochem., 2011, 75, 633-637.

57 C. P. Racz, S. Santa, M. Tomoaia-Cotisel, G. Borodi, I. Kacso, A. Pirnau and I. Bratu, J. Incl. Phenom. Macrocycl. Chem., 2013, 76, 193199.

58 M. R. Caira, S. A. Bourne and B. Mzondo, Molecules, 2017, 22, 866.

59 N. Ikuta, H. Sugiyama, H. Shimosegawa, R. Nakane, Y. Ishida, Y. Uekaji, D. Nakata, K. Pallauf, G. Rimbach, K. Terao and S. Matsugo, Int. J. Mol. Sci., 2013, 14, 3639-3655.

60 A. Celebioglu and T. Uyar, J. Agric. Food Chem., 2019, 67, 13093-13107.

61 H. Lees, M. Vaher and M. Kaljurand, Electrophoresis, 2017, 38, 1075-1082.

62 P. Jõul, M. Vaher and M. Kuhtinskaja, Chemosphere, 2018, 198, 460-468.

63 Ł. Dobrzycki, P. Socha, A. Ciesielski, R. Boese and M. K. Cyrański, Cryst. Growth Des., 2019, 19, 1005-1020.

64 S. Mecozzi and Jr. Rebek Julius, Chem. Eur. J., 1998, 4, 1016-1022.

65 M. Dračínský, C. S. Hurtado, E. Masson and J. Kaleta, Chem. Commun., 2021, 57, 2132-2135. 